\title{
Bronchial carcinoid tumors: Surgical management and long-term outcome
}

\author{
Pier Luigi Filosso, MD \\ Ottavio Rena, MD \\ Giovanni Donati, MD \\ Caterina Casadio, MD \\ Enrico Ruffini, MD \\ Esther Papalia, MD \\ Alberto Oliaro, MD \\ Giuliano Maggi, MD
}

From the Department of Thoracic Surgery, University of Torino, San Giovanni Battista Hospital, Torino, Italy.

Read at the Eighty-first Annual Meeting of The American Association for Thoracic Surgery, San Diego, Calif, May 6-9, 2001.

Received for publication May 22, 2001; revisions requested July 3, 2001; revisions received July 23, 2001; accepted for publication Aug 30, 2001

Address for reprints: Pier Luigi Filosso, MD, Department of Thoracic Surgery, University of Torino, San Giovanni Battista Hospital, via Genova, 3, 101216, Torino, Italy (E-mail: pierluigifilosso@tiscalinet.it).

J Thorac Cardiovasc Surg 2002;123:303-9

Copyright (C) 2002 by The American Association for Thoracic Surgery

$0022-5223 / 2002 \$ 35.00+0 \quad \mathbf{1 2 / 6 / 1 1 9 8 8 6}$

doi: $10.1067 / \mathrm{mtc} .2002 .119886$
Objective: We sought to determine the variables influencing long-term survival of patients treated for bronchial carcinoid tumors.

Methods: A retrospective, mono-institutional review of patients subjected to surgical treatment since 1977 was conducted.

Results: Over 22 years, 126 patients with a final histologic diagnosis of bronchial carcinoid tumors were assessed for surgery. The group comprised 72 men (57\%) and 54 women $(43 \%)$ with a mean age at presentation of $47 \pm 16$ years (range 1177 years). Symptoms were present in $65(53 \%)$ patients. Operations included lobectomy or bilobectomy in 88 (with 4 bronchoplastic procedures), pneumonectomy in 15 , segmentectomy in 3 , wedge resection in 16, and bronchial sleeve resection in 3 patients. One patient $(0.7 \%)$ died in the perioperative period. Eighty-two patients (65\%) had typical and $44(35 \%)$ had atypical carcinoid tumors. Postoperative staging was complete for 113 of 126 patients (13 patients did not undergo lymphadenectomy): 90 patients had stage I disease, 6 had stage II, 15 had stage III, and 2 had stage IV disease. A typical subtype was stage I in 70 and more advanced (IIIV) in 5, whereas an atypical subtype was stage I in 20 and more advanced in 18 ( $P$ $<.05$ ). Mean follow-up was $99 \pm 73$ months (range 6-282 months) during which 19 $(15 \%)$ patients died (12 of recurrent disease). Recurrent tumor developed in 4 $(5.5 \%)$ of 72 patients affected by typical subtypes and $8(19.5 \%)$ of 41 by atypical subtypes with complete follow-up. Overall survival at 15 years was $74 \%$; survival related to histologic type and nodal status at 15 years was significant $(P<.05)$.

Conclusions: Biologic behavior and prognosis for bronchial carcinoid tumors are better than for other lung cancers. Surgical treatment requires radical excision and lymph node sampling. Survival and long-term outcome are significantly related to the histologic type, nodal status, and pathologic stage.

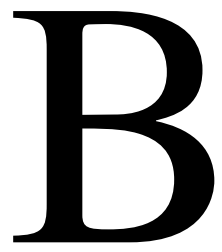

ronchial carcinoid tumors are rare, constituting less than $2 \%$ of all pulmonary neoplasms ${ }^{1} ; 90 \%$ of them are confined to the bronchus and only $10 \%$ have regional lymph node involvement. ${ }^{2}$

The tumors occur at any age, have the same incidence in both sexes, and are characterized by mainly slow endobronchial growth., Many patients are free of symptoms, the tumor often being an incidental radiologic finding. Smoking seems to predispose to the development of these tumors, as it does to the development of bronchial carcinoma. ${ }^{5-7}$

The Journal of Thoracic and Cardiovascular Surgery • Volume 123, Number 2303 
In the recent classification of lung tumors by the World Health Organization (WHO) and International Association for the Study of Lung Cancer (IASLC), bronchial carcinoids are considered part of the neuroendocrine tumor spectrum (typical carcinoid, atypical carcinoid, large cell neuroendocrine carcinoma, and small cell carcinoma), and typical and atypical carcinoids are grouped together. ${ }^{8}$ This is the direct consequence of the fact that typical and atypical carcinoid tumors are more closely related clinically than other neuroendocrine tumors and share similar biologic markers. ${ }^{9}$

\section{Patients and Methods}

We retrospectively reviewed our experience with the management of both typical and atypical carcinoids to analyze recurrence rates, survival, and factors that might influence these variables.

Between 1977 and 1999, 126 patients with a final histologic diagnosis of bronchial carcinoid tumor were assessed for surgery; in 81 of them, diagnosis and treatment were accomplished during the past 11 years. Evaluation comprised history and physical examination, posteroanterior and lateral chest radiographs, and computed tomographic (CT) scans of the chest and upper abdomen (including liver and adrenal glands). Bone scintiscans and brain CT scans were performed in cases of bone pain or neurologic symptoms or signs. Performance status was assessed by the Karnofsky scale. ${ }^{10}$ Pulmonary function tests including circulation of single breath diffusing capacity for carbon monoxide were performed routinely.

All patients had a preoperative examination with a fiberoptic bronchoscope, and in some cases endoscopic biopsy was performed. Fine-needle aspiration biopsy was performed in peripherally located tumors. At surgery, all specimens resected, including hilar and mediastinal lymph nodes, were sent for histologic examination; tumors were classified according to the current WHO/IASLC criteria for neuroendocrine tumors. ${ }^{8}$ Typical carcinoids are defined as tumors greater than $5 \mathrm{~mm}$ in diameter, with carcinoid morphology and less than 2 mitoses per $2 \mathrm{~mm}^{2}$, and lacking necrosis. Tumors with a mitosis rate of 2 or more but less than 10 per $2 \mathrm{~mm}^{2}$, with or without necrosis, were classified as atypical. $^{8-11}$

Patients were followed up for periods ranging from 6 to 282 months (mean $99 \pm 73$ months). We analyzed the influence of tumor site, carcinoid morphology, lymph node involvement, and the extent of resection on the outcome of surgery.

\section{Results}

\section{Clinical Presentation}

The group comprised $72(57 \%)$ male and 54 (43\%) female patients; age at presentation ranged from 11 to 77 years (mean $47 \pm 16$ years). Patients with atypical carcinoid were, on average, 9.5 years older at presentation than those with typical subtype (mean 55 vs 45.5 years, respectively; $P<$ $.05)$. Symptoms were present in 65 (52\%) patients: hemoptysis $(\mathrm{n}=27)$, cough $(\mathrm{n}=26)$, recurrent pulmonary infection $(n=21)$, fever $(n=15)$, chest discomfort and chest pain $(\mathrm{n}=14)$, wheezing $(\mathrm{n}=11)$, and dyspnea $(\mathrm{n}=9)$. Eighteen
(14.2\%) patients had been treated for asthma for up to 3 years before the discovery of the tumor. Sixty-one (47\%) patients were asymptomatic at presentation.

\section{Investigations}

All patients underwent chest radiographs, chest CT scans, and preoperative examination with a fiberoptic bronchoscope. The tumors could be visualized at bronchoscopy in 103 patients, and these were classified as central tumors; the other 23 tumors were classified as peripheral tumors.

\section{Localization of the Tumor}

The tumor was in the right lung in $77(61 \%)$ patients and in the left lung in 49. The 103 (82\%) tumors that were centrally located arose in the following sites: right main bronchus, 5; left main bronchus, 11; intermediate bronchus, 20; middle lobe bronchus, 9; right upper lobe bronchus, 15; right inferior lobe bronchus, 16; left upper lobe bronchus, 12; and inferior left bronchus, 15. The other 23 tumors were peripherally located.

Operations included 15 pneumonectomies ( 9 of them performed before the 1990s), 64 standard lobectomies (14 right superior, 9 middle, 16 right inferior, 11 left superior, 14 left inferior), 4 sleeve lobectomies (1 right superior, 2 middle, 1 left superior), 17 bilobectomies, 3 bronchial sleeve resections, 3 segmentectomies, and 16 wedge resections.

\section{Pathology}

In 70 patients of 82 with a definitive diagnosis of typical carcinoid, preoperative bronchoscopy allowed the direct vision of the centrally located tumor. Endoscopic biopsy was carried out in 47 cases to assess the histologic diagnosis: 26 were considered typical carcinoid, 2 atypical carcinoid, 9 non-small cell lung cancer, and 10 were nondiagnostic. Nine patients who had non-small cell lung cancer according to preoperative bronchoscopic biopsy (before the 1990s) were subjected to pneumonectomy. The definitive diagnosis of typical carcinoids retrospectively indicated that sleeve resections of the left main stem bronchus could have been performed in 6 patients and of the right main stem bronchus in 3 patients.

Preoperative bronchoscopic biopsy tissue was available in 28 of 44 patients with a definitive diagnosis of atypical carcinoid: the specimens were interpreted as showing atypical carcinoid in 14 patients, typical carcinoid in 8, non-small cell lung cancer in 2 , and were nondiagnostic in 4 patients. Fine-needle aspiration biopsy was carried out in 9 of 23 patients with peripherally located tumors. Correct diagnosis with reference to the subtype of neuroendocrine tumor was obtained for 50\% of typical carcinoids and for 66\% of atypical ones: the remaining cases were misinterpreted. All preoperative specimens were revised after the definitive 
TABLE 1. Typical and atypical bronchial carcinoids subdivided by pathologic staging

\begin{tabular}{lcc}
\hline & Typical & Atypical \\
\hline Stage I & 70 & 20 \\
Stage II & 2 & 4 \\
Stage III & 3 & 12 \\
Stage IV & 0 & 2 \\
Total & 75 & 38
\end{tabular}

TABLE 2. Overall long-term survival of patients with bronchial carcinoids

\begin{tabular}{ll}
\hline Duration of survival & \\
\hline 5 years & $89 \%$ \\
10 years & $79 \%$ \\
15 years & $74 \%$ \\
\hline
\end{tabular}

Mean $8.3 \pm 6.14$ years (range $0-23$ years).

diagnosis, and in each case the cause of the incorrect diagnosis was found to be a misinterpretation of the cellular pleomorphism. Postoperative staging was complete for 113 of 126 patients (13 patients did not undergo lymph nodal sampling). Seventy-five (65\%) of 113 tumors were considered typical carcinoids, with $6(8 \%)$ showing lymph node localization ( 2 in an N1 and 4 in an N2 position). Because this is a relatively high percentage for typical carcinoids, these cases were resubmitted to our pathologists' evaluation, and the possibility that these were atypical carcinoids was excluded (every case demonstrated less than 2 mitotic figures per high-power field). In no case were the lymph nodes contiguous with the primary mass. Each N1 cases had hilar lymph node metastases associated with upper lobe tumors. N2 cases had subcarinal lymph node metastases related to 3 inferior lobe tumors and 1 middle lobe tumor.

Fourteen (36.8\%) of 38 patients with atypical carcinoid tumors had nodal deposits, 7 in N1 nodes and 7 with N2 disease. In each case of $\mathrm{N} 2$ disease the diagnosis was made postoperatively, so that only adjuvant radiation therapy was administered.

Stage was more advanced among patients with atypical histologic subtype regardless of the means of categorization $(P<.05)$. Relative stages for typical and atypical carcinoids, according to pathologic evaluation of the operative samples, are shown in Tables 1, 2, 3, and 4. Typical subtype was stage I in 70 and more advanced (stage II-IV) in 5 patients, whereas atypical subtype was stage I in 20 and more advanced in 18 patients $(P<.05)$.

Follow-up was complete until death or within at most 6 months of analysis in all patients with complete staging: 113
TABLE 3. Long-term survival related to the histologic subtype

\begin{tabular}{lcc}
\hline Duration of survival & Typical carcinoid & Atypical carcinoid \\
\hline 5 years & $97 \%$ & $77 \%$ \\
10 years & $93 \%$ & $52 \%$ \\
15 years & $84 \%$ & $52 \%$
\end{tabular}

Log-rank test: $P=.0004$; Cox test: $P=.0014$.

TABLE 4. Long-term survival related to the nodal status

\begin{tabular}{lcc}
\hline Duration of survival & $\mathbf{N +}$ & $\mathbf{N}-$ \\
\hline 5 years & $85 \%$ & $92 \%$ \\
10 years & $52 \%$ & $87 \%$ \\
15 years & $52 \%$ & $80 \%$ \\
\hline
\end{tabular}

Log-rank test: $P=.021$; Cox test: $P=.011$.

(89.8\%) of 126 patients. The mean follow-up was $99 \pm 73$ months (median 84 months, range 6-282 months). Recurrent cancer developed in $4(5.5 \%)$ of 72 patients with typical and $8(19.5 \%)$ of 41 with atypical subtype $(P<.05)$. Four patients had intrathoracic recurrence after resection of atypical tumor. The disease recurred after a median of 3.5 years (range 2-5.5 years). Because reoperation was not feasible (poor pulmonary function tests in 3 patients and advanced age in 1 patient), these 4 patients were subjected to chemotherapy and radiation therapy. The median survival for these patients was 3 years (range 2.5-5 years). Eight patients with recurrent atypical carcinoid had bone and brain metastases, associated with intrathoracic recurrence in 1 , bone metastases in 1 , and liver metastases in 6 patients, a median of 2.5 years (range 1.5-4 years) after the first operation. Five patients (affected by liver or bone metastases) were subjected to octreotide or chemotherapy. The median survival after diagnosis of the recurrent disease was 2 years (range 1.5-3 years). All patients with recurrent disease died. The incidence of tumor-related deaths is $12(10.6 \%)$ of 113 patients.

Overall survival at 15 years is $74 \%$ (Figure 1). A multivariate Cox proportional hazards analysis of survival was performed with the covariates histologic subtype (Figure 2) and nodal status (Figure 3).

\section{Discussion}

Bronchial carcinoids have gained importance for thoracic surgeons, in particular whether to regard them as malignant or benign tumors. ${ }^{3}$ The WHO/IASLC classification ${ }^{8}$ includes typical and atypical carcinoids in the spectrum of neuroendocrine tumors but groups them separately from 


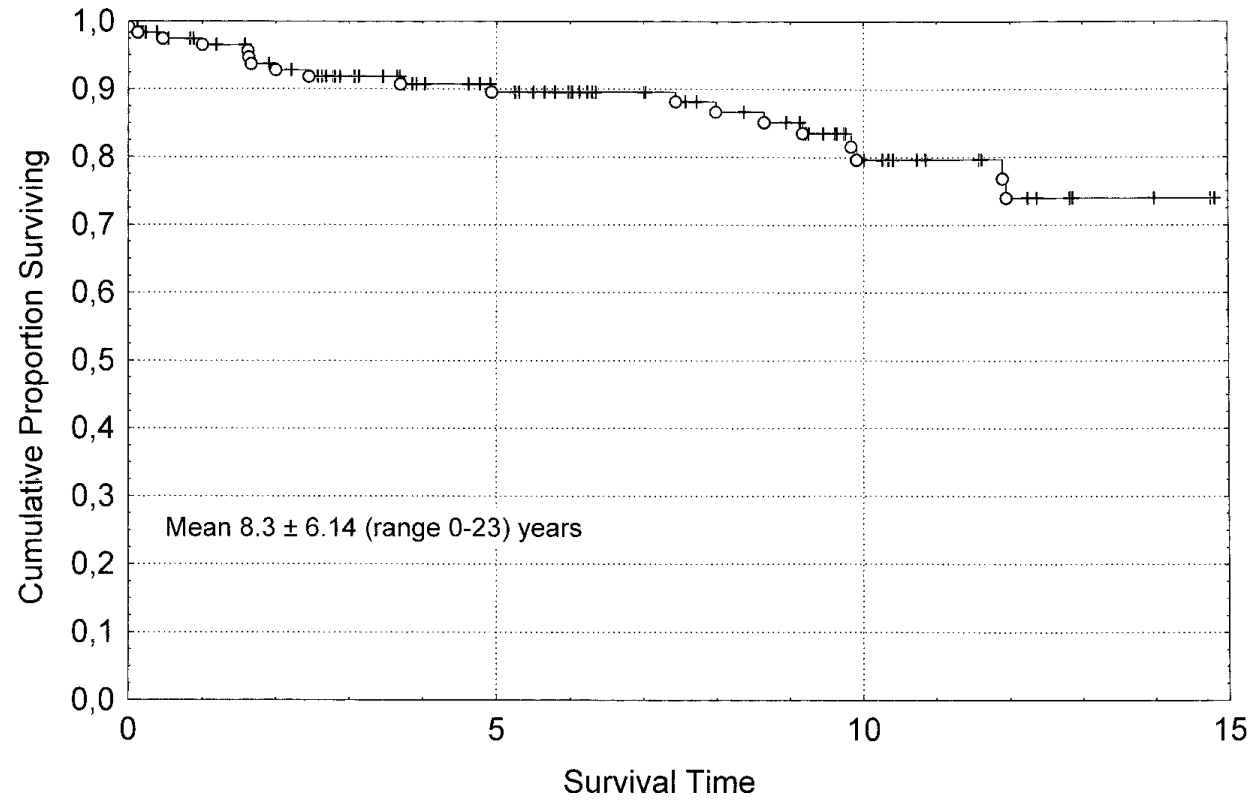

Figure 1. Overall long-term survival.

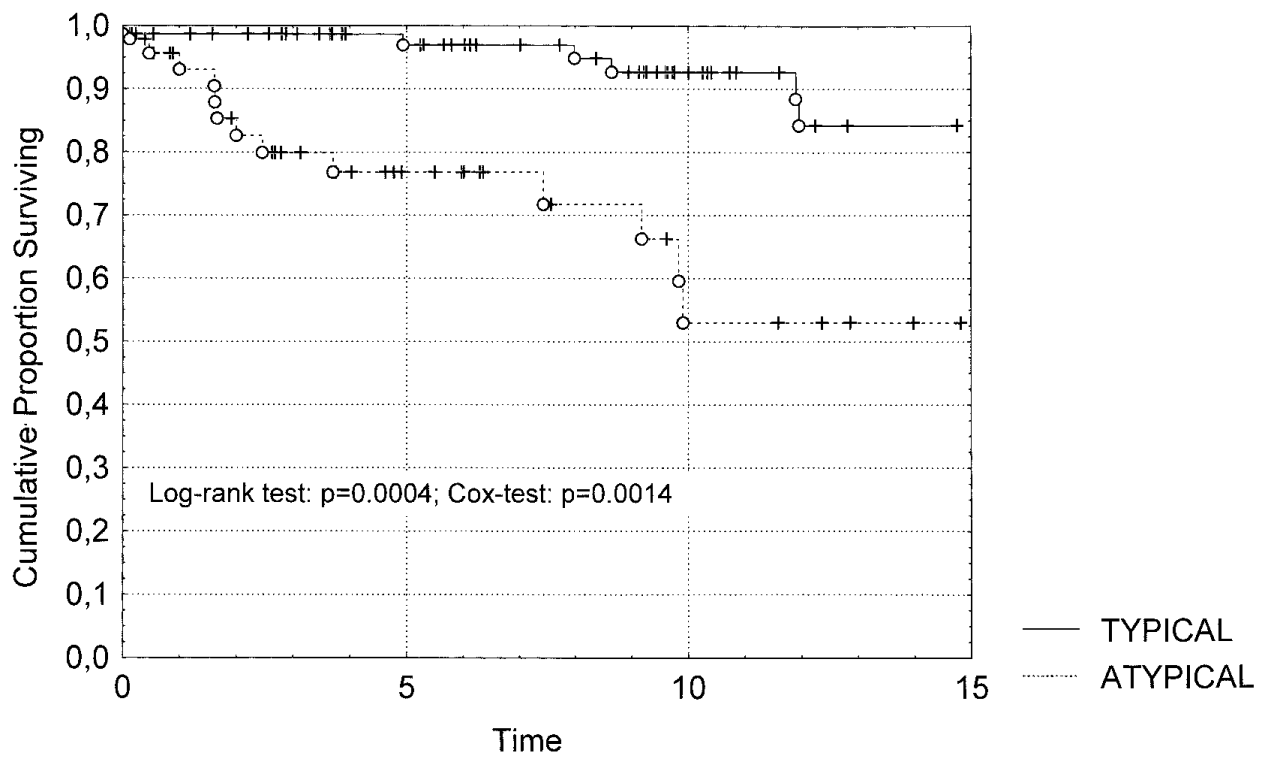

Figure 2. Cumulative proportion surviving (Kaplan-Meier). Long-term survival related to histologic subtype.

large cell neuroendocrine carcinoma and small cell lung carcinoma on the basis of closer histologic and biologic interrelationships. ${ }^{6,11}$ They are low-grade malignant tumors, which can be locally invasive or spread to lymph nodes, but distant metastases are rare.

The prevalence of these tumors is almost equal in male and female patients. ${ }^{12}$ The mean age of our patients at pre- sentation was $47 \pm 16$ years (range 11-77 years), which is in line with other reports. ${ }^{4,13,14}$ As mentioned by El Jamal and colleagues, ${ }^{13}$ patients having atypical carcinoid tumors are significantly older at presentation than are patients with typical tumors.

The influence of smoking on the pathogenesis of carcinoid tumors is still debated. Two thirds of patients were 


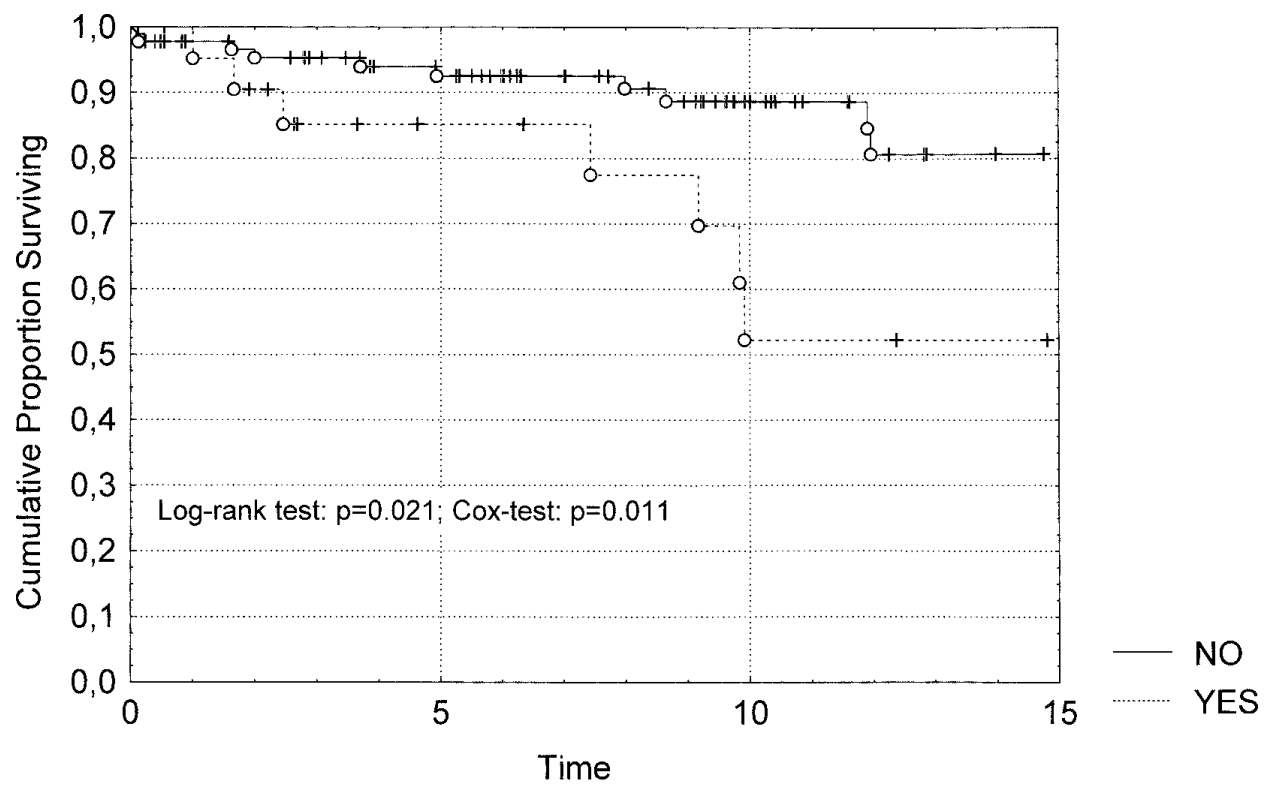

Figure 3. Cumulative proportion surviving (Kaplan-Meier). Long-term survival related to nodal involvement.

smokers or ex-smokers, as some authors mentioned. ${ }^{7} \mathrm{We}$ and El Jamal's group ${ }^{13}$ noted that the mean age at the diagnosis of atypical carcinoid is significantly higher than that of patients affected by typical carcinoid and is similar to that of patients with lung carcinoma that is related to cigarette smoking. Detection of a bronchial carcinoid may follow a variety of pulmonary symptoms, or the tumors may be found on a routine chest radiograph.

Bronchoscopy plays a big role in the diagnosis of carcinoids: in the majority of cases the tumor is centrally located and visible at endoscopic evaluation, as other authors have described. ${ }^{13,15}$ Some authors found bleeding to occur in two thirds of their patients and some advise against biopsy when carcinoid is suspected $^{6,16,17}$; other authors disagreed, maintaining that bronchoscopic biopsy significantly increases the diagnostic yield without adding morbidity. ${ }^{18}$ In our experience, no troublesome bleeding was reported after endoscopic biopsy. The main problem was the difficulty in obtaining an accurate diagnosis in carcinoid tumors. Endoscopic sampling suggested the diagnosis of carcinoid in $67 \%$ of cases, and the correct subtype of the tumor was detected in $53 \%$. Of 75 bronchoscopic biopsies, 35 were not diagnostic or yielded an incorrect diagnosis: in 11 of the 35 , non-small cell lung cancer was preoperatively diagnosed and then pneumonectomy was performed. Nine of 11 were postoperatively diagnosed as typical carcinoids, and pneumonectomy clearly was unnecessary inasmuch as all patients could have been successfully treated by a bronchial sleeve resection. The accuracy of fine-needle aspiration biopsy suggests the correct diagnosis in peripherally located tumors was about $55 \%$. It is not surprising that the pathologist should have difficulty in differentiating typical from atypical carcinoid tumors, because such features as increased mitotic rate and necrosis may not be present in a biopsy sample.

Most tumors are localized and the likelihood of local recurrence after resection is small. This is attested to by a 15 -year survival of $80 \%$ in patients with localized disease and $52 \%$ in patients with regionally advanced or metastatic disease in our study.

The assessment of the biologic behavior of bronchial carcinoid tumors is not always accurate. Atypical carcinoid tumors present more often at a more advanced stage, are more likely to recur, and are associated with lower 5- and 10 -year survivals than are typical carcinoids. These findings reveal the need for more accurate differentiation between histologic subtypes. Strict application of classification criteria and perhaps revision of classification criteria are mandatory to allow distinction between typical and atypical subtypes that could influence surgical management.

During recent years, immunohistochemical analysis, measurement of DNA ploidy, and assessment of chromosomal deletions $(11 \mathrm{q}, 3 \mathrm{p})$ have been proposed, but none of these has been shown to be useful preoperatively or intraoperatively to guide selection of surgical management. ${ }^{19-21}$ Actually, the only reliable factors for assessing aggressiveness are the tumor stage and its histologic subtype.

Preoperative radiologic evaluation and histologic type are mandatory in selecting the extent of surgical resection. Newer modalities for staging bronchial carcinoid tumors 
that have recently been introduced include positron emission tomography and octreotide scintigraphy. Positron emission tomographic imaging of bronchial carcinoid tumors demonstrates lower uptake than non-small cell lung cancers, suggesting that the process is benign and that staging of regional lymph nodes might be unreliable. ${ }^{22}$ Scintigraphy with ${ }^{111}$ In-octreotide has demonstrated reliable uptake in primary tumors and the ability to detect early recurrences and metastases even in asymptomatic patients, suggesting that it might be a useful tool for routine staging in the future. ${ }^{23}$ At present, however, decisions regarding appropriate therapy for patients with bronchial carcinoid tumors are made on the basis of histologic findings, clinical staging with bronchoscopy and CT, and in some instances intraoperative lymph node staging.

The current surgical management of bronchial carcinoids is influenced by the recurrence rates and survival patterns we and others have observed. ${ }^{1,6,9,13,14,16-18,24,25}$ In patients with centrally located typical carcinoid tumor of the lung, we think that bronchial sleeve resection or sleeve lobectomy should be considered, when possible, because local recurrence is rare and survival is excellent. Despite the low local recurrence rate, early-stage typical carcinoids should be considered to be low-malignancy neoplasms and should be managed by an anatomic resection when peripherally located (lobectomy if pulmonary function tests are adequate or segmentectomy in other cases) to secure the least risk of recurrence. On the other hand, local recurrence rate and long-term survival are both unfavorably affected by the finding of atypical subtype: if this histologic subtype is identified, a more extensive surgical approach (such as lobectomy or pneumonectomy) associated with lymph node dissection is mandatory.

\section{References}

1. Chughtai T, Morin J, Sheiner N, Wilson J, Mulder D. Bronchial carcinoid: twenty years' experience defines selective surgical approach. Surgery. 1997;122:801-8.

2. Martini N, Zaman M, Banis M, Burt M, McCormack P, Rush V, et al. Treatment and prognosis in bronchial carcinoid involving regional lymph nodes. J Thorac Cardiovasc Surg. 1994;107:1-7.

3. Goldstraw P, Lamb D, McCormack R, Walbaum P. The malignancy of bronchial adenoma. J Thorac Cardiovasc Surg. 1976;72:309-14.

4. Hasleton P. Histopathology and prognostic factors in bronchial carcinoid tumours. Thorax. 1994;49:s56-62.

5. Martenson H, Botcher G, Hambraeus G, Sundler F, Willen H, Nobin A. Bronchial carcinoid: an analysis of 91 cases. World J Surg. 1987; 11:56-64.

6. Marty-Anè C, Costes V, Pujol J, Alauzen M, Baldet P, Mary H. Carcinoid tumors of the lung: Do atypical features require aggressive management? Ann Thorac Surg. 1995;59:78-83.

7. Foudarakis M, Fournel P, Bungard G, Borros D, Boucheron S, Siakafas N, et al. Bronchial carcinoid: a review of 22 cases. Oncology. 1996;53:153-8.

8. Travis WD, Corrin B, Shimosato Y, Brambilla E. The histological typing of lung and pleural tumours. WHO/IASLC classification of lung and pleural tumours, 3rd ed. Berlin: Springer Verlag; 1999.

9. Travis W, Rush V, Flieder D, Falk R, Fleming M, Gal A, et al. Survival analysis of 200 pulmonary neuroendocrine tumors with classification of criteria for atypical carcinoid and its separation from typical carcinoid. Am J Surg Pathol. 1995;22:934-44.

10. Stanley KE. Prognostic factors for survival in patients with inoperable lung cancer. J Natl Cancer Inst. 1980;65:25-32.

11. Paladagu R, Benfield J, Pak H, Ross R, Teplitz R. Bronchopulmonary Kulchitsky cell carcinomas. Cancer. 1985;55:1303-11.

12. Okike N, Bernatz P, Woolner L. Carcinoid tumors of the lung. Ann Thorac Surg. 1976;22:207-12.

13. El Jamal M, Nicholson AG, Goldstraw P. The feasibility of conservative resection for carcinoid tumours: Is pneumonectomy ever necessary for uncomplicated cases? Eur J Cardiothorac Surg. 2000;18:301-6.

14. Ferguson MK, Landreneau RJ, Hazelrigg SR, Altorky NK, Naunheim $\mathrm{KS}$, Zwischenberger JB, et al. Long-term outcome after resection for bronchial carcinoid tumours. Eur J Cardiothorac Surg. 2000;18:156-61.

15. Okike N, Bernatz P, Payne W, Woolner L, Leonard P. Bronchoscopic procedures in treatment of carcinoid tumors of the tracheobronchial tree. J Thorac Cardiovasc Surg. 1978;76:281-91.

16. Todd T, Cooper J, Weisberg D, Delarue N, Pearson F. Bronchial carcinoid tumors: twenty years' experience. $J$ Thorac Cardiovasc Surg. 1980;79:32-6

17. McCoughan BC, Martini N, Bains M. Bronchial carcinoids: review of 124 cases. J Thorac Cardiovasc Surg. 1985;89:8-17.

18. Rea F, Binda R, Spreafico G, Calabrò F, Bonavina L, Cipriani A, et al. Bronchial carcinoids: a review of 60 patients. Ann Thorac Surg. 1989;47:412-4.

19. Padberg BC, Woenckhaus J, Hilger G, Beccu L, Jochum W, Range U, et al. DNA cytophotometry and prognosis in typical and atypical bronchopulmonary carcinoids. Am J Surg Pathol. 1996;20:815-22.

20. Kovatich A, Friedland DM, Druck T, Hadaczek P, Huebner K, Comis $\mathrm{RL}$, et al. Molecular alterations to human chromosome $3 \mathrm{p}$ loci in neuroendocrine lung tumors. Cancer. 1998;83:1109-17.

21. Walch AK, Zitzelsberger HF, Aubele MM, Mattis AE, Bauchinger M, Candidus S, et al. Typical and atypical carcinoid tumors of the lung are characterized by $11 \mathrm{q}$ deletions as detected by comparative genomic hybridization. Am J Pathol. 1998;153:1089-98.

22. Erasmus JJ, McAdams HP, Patz Jr EF, Coleman RE, Ahuja V, Goodman PC. Evaluation of primary pulmonary carcinoid tumors using FDG PET. Am J Roentgenol. 1998;170:1369-73.

23. Musi M, Carbone RG, Bertocchi C, Cantalupi DP, Michetti G, Pugliese C, et al. Bronchial carcinoid tumors: a study on clinicopathological features and role of octreotide scintigraphy. Lung Cancer. 1998;22:97-102.

24. Hurt R, Bates M. Carcinoid tumours of the bronchus: a 33 year experience. Thorax. 1984;39:617-23.

25. Stamatis G, Freitag L, Greschuchna D. Limited and radical resection for tracheal and bronchopulmonary carcinoid tumour. Eur $J$ Cardiothorac Surg. 1990;4:527-32.

\section{Discussion}

Dr Mark S. Allen (Rochester, Minn). Carcinoid tumors are a unique type of malignant pulmonary disease. They are rare, comprising less than $5 \%$ of all primary pulmonary neoplasms. Most of them arise in the bronchial airways. Usually they are classified as a typical carcinoid, a less virulent variety, versus an atypical variety, a more virulent type. However, both types of carcinoids are malignant and should be treated as such. This series represents a large number of patients who have undergone surgical resection for bronchial carcinoid tumors. This series is among the largest in the literature and shows characteristics that others have seen. The male/female distribution is nearly equal, no clear association with cigarette smoking is identified, and long-term survival after complete resection is relatively good.

Since pulmonary carcinoids are usually located in the central bronchial tree, they are readily visualized by bronchoscopy. Dr Filosso, you say that you performed a preoperative bronchoscopic 
examination in all patients, but I am puzzled about why you obtained biopsy specimens in $98(77 \%)$ of the 126 patients. I do not think a biopsy is necessary in the majority of patients with a bronchial carcinoid once the bronchoscopic examination has disclosed a resectable lesion. The pathologic distinction between typical or atypical is not really very important, because regardless of what the pathologic examination shows, surgical resection is the proper management. Furthermore, as you have shown, the pathology results from the biopsy are often misleading. Do you think biopsies are appropriate in all bronchial tumors, and did you have any difficulty with bleeding in any of the 98 biopsies?

My second question relates to the operation you performed on your patients. You stated that $82 \%$ of the patients had a centrally located lesion. Eleven patients had a left main stem bronchial lesion, 5 lesions were in the right main stem bronchus, and 20 were in the bronchus intermedius. Yet, only $7(5 \%)$ of the 126 patients had a sleeve resection, and $15(12 \%)$ patients required a pneumonectomy. Do you think that sleeve resection is not a good operation for a bronchial carcinoid? Why was the number of sleeve resections not higher? I think that a lung-conserving operation is best for a patient with a carcinoid if it is feasible.

Finally, in your analysis of the outcome, the only factors that influenced survival were the histologic subtype and nodal disease. Did you examine any molecular markers, such as DNA ploidy, p53 levels, or chromosome deletions to determine whether these affected survival?

Dr William H. Warren (Chicago, Ill). The literature on lowgrade neuroendocrine tumors has been needlessly confused by persisting in lumping typical and "atypical" carcinoids as if they were one entity with two different minor subtypes. Thus, when we find their prognoses are different, we conclude that they should be distinguished again. These are two separate tumors, and I think it is time we start discussing them as such from the beginning.
I have two questions: First, 5 patients with typical bronchial carcinoids were found to have nodal metastases. Did that influence survival? Second, if nodal metastases were found, would you consider giving chemotherapy, and, if so, what type? In our series of over 150 typical bronchial carcinoids at Rush Presbyterian-St Luke's Medical Center, we have found 7 patients with N1 or N2 nodal metastases, but this has not seemed to influence the prognosis without any form of adjuvant therapy.

Dr Filosso. I agree with Dr Allen about the opportunity to perform sleeve lobectomies in carcinoid tumors, when possible. Pneumonectomies and bilobectomies were performed, in our series, when the lung was destroyed by long-term infections. In fact, some of our patients were treated for asthma for 2 or 3 years before a bronchial carcinoid was diagnosed. In the last period of our experience, the number of pneumonectomies and bilobectomies was dramatically reduced thanks to correct preoperative diagnoses. We performed bronchoscopy in all patients with a centrally located tumor, and we had no experience of troublesome bleeding during these procedures.

The possibility to use molecular markers, such as p53 or Ki67, could help us to determine the outcome of neuroendocrine tumors, but at the department of pathology of our hospital these procedures are not yet routinely used. I have, on the contrary, an interesting experience with ${ }^{111}$ In-diethylenetriamine pentaacetate-pentetreotide scintigraphy (OctreoScan), used in both the preoperative and the follow-up studies. Mediastinal lymph node metastases and recurrences of resected carcinoids were correctly diagnosed by this procedure, and in selected cases, I treated recurrences (not resectable) or distant metastases with "cold" octreotide, with success.

To Dr Warren: We had not observed a significant decrease in long-term survival of $\mathrm{N} 2$ typical carcinoids. We do not perform chemotherapy in these patients: we prefer radiation therapy.

\section{Targeted}

The Journal of Thoracic and Cardiovascular Surgery gives you two tables of contents.

The condensed table of contents tells you at a glance what topics and authors are presented each month. The expanded table of contents gives you a brief abstract of each article. You select only those articles of most interest to you for further reading. 